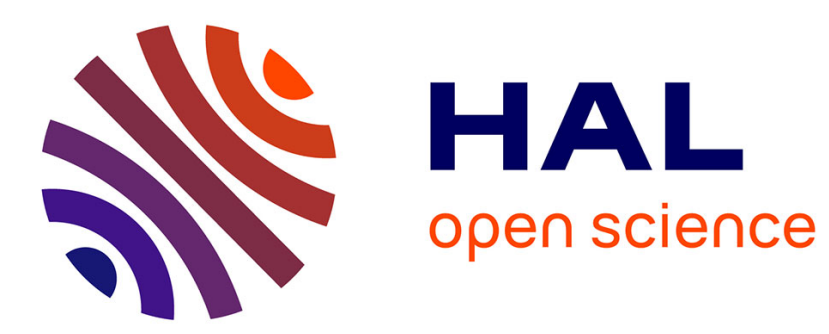

\title{
Homomorphisms of 2-edge-colored triangle-free planar graphs
}

Pascal Ochem, Alexandre Pinlou, Sagnik Sen

\section{To cite this version:}

Pascal Ochem, Alexandre Pinlou, Sagnik Sen. Homomorphisms of 2-edge-colored triangle-free planar graphs. Journal of Graph Theory, 2017, 85 (1), pp.258-277. 10.1002/jgt.22059 lirmm-01376130

\section{HAL Id: lirmm-01376130 \\ https://hal-lirmm.ccsd.cnrs.fr/lirmm-01376130}

Submitted on 4 Oct 2016

HAL is a multi-disciplinary open access archive for the deposit and dissemination of scientific research documents, whether they are published or not. The documents may come from teaching and research institutions in France or abroad, or from public or private research centers.
L'archive ouverte pluridisciplinaire $\mathbf{H A L}$, est destinée au dépôt et à la diffusion de documents scientifiques de niveau recherche, publiés ou non, émanant des établissements d'enseignement et de recherche français ou étrangers, des laboratoires publics ou privés. 


\title{
Homomorphisms of 2-edge-colored triangle-free planar graphs ${ }^{1}$
}

\author{
Pascal Ochem \\ Université de Montpellier, LIRMM, CNRS, France. \\ Alexandre Pinlou \\ Université Paul-Valery Montpellier, LIRMM, CNRS, France. \\ Sagnik Sen \\ Indian Statistical Institute, Kolkata, India.
}

\begin{abstract}
In this paper, we introduce and study the properties of some target graphs for 2-edge-colored homomorphism. Using these properties, we obtain in particular that the 2-edge-colored chromatic number of the class of triangle-free planar graphs is at most 50. We also show that it is at least 12 .
\end{abstract}

Key words: 2-edge-colored graph, Graph homomorphism, Discharging method.

\section{Introduction}

Graph homomorphisms and proper graph colorings are closely related. Indeed, the homomorphisms $\varphi: G \rightarrow K_{k}$ are the $k$-colorings of $G$. This explains that homomorphisms of $G$ to $H$ are usually called $H$-colorings of $G$ (we also say $G$ is $H$-colorable) and that the vertices of such graphs $H$ are called colors. The chromatic number of $G$ is defined as the minimum number of colors $k$ such that $G$ admits a $k$-coloring; it can be equivalently defined as the minimum order of a graph $H$ such that $G \rightarrow H$.

Email addresses: Pascal.Ochem@lirmm.fr (Pascal Ochem), Alexandre.Pinlou@lirmm.fr (Alexandre Pinlou), sen007isi@gmail.com (Sagnik Sen).

URLs: http://www.lirmm.fr/ ochem (Pascal Ochem), http://www.lirmm.fr/ ${ }^{\sim}$ pinlou (Alexandre Pinlou).

1 This work was partially supported by the ANR grant EGOS 12-JS02-002-01 and by the PEPS grant HOGRASI. 


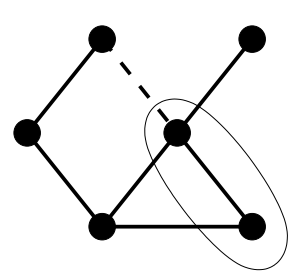

(a)

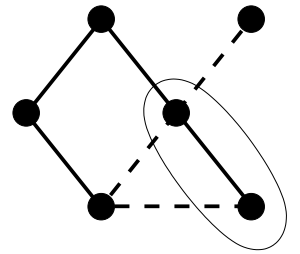

(b)

Fig. 1. Two equivalent 2-edge-colored graphs.

In this paper, we propose to study homomorphisms of 2-edge-colored graphs and the related notion of coloring. They have been already studied as a way of extending classical results in graph coloring such as Hadwiger's conjecture. Guenin [5] introduced the notion of switching homomorphism for its relation with a well known conjecture of Seymour. In 2012, this notion has been further developed by Naserasr et al. [9] as this theory captures a number of well known conjectures which can be reformulated using the definitions of switching homomorphism. In this paper, we study 2-edge-colored graph homomorphisms and switching homomorphisms for themselves.

A 2-edge-colored graph $(G, s)$ is a simple graph $G$ with a signature $s: E(G) \rightarrow$ $\{-1,+1\}$ assigning a negative or positive sign to every edge of $G$. In the figures, negative edges are drawn with dashed edges. Figure 1(a) gives an example of 2-edge-colored graph. Switching a vertex $v$ of a 2-edge-colored graph $(G, s)$ corresponds to giving the opposite sign to the edges incident to $v$. Given a 2edge-colored graph $(G, s)$ and a set of vertices $X \subseteq V(G)$, the graph obtained from $(G, s)$ by switching every vertex in $X$ is denoted by $\left(G, s^{(X)}\right)$. Let $\partial(X)$ denote the edge cut between $X$ and $G \backslash X$. Notice that $\left(G, s^{(X)}\right)$ is equivalently obtained by changing the sign of all the edges in $\partial(X)$.

Two 2-edge-colored graphs $\left(G, s_{1}\right)$ and $\left(G, s_{2}\right)$ are switching equivalent if we can obtain $\left(G, s_{1}\right)$ from $\left(G, s_{2}\right)$ by switching a set of vertices of $\left(G, s_{2}\right)$, i.e., $s_{2}=s_{1}^{(X)}$ with $X \subseteq V(G)$. If $\left(G, s_{1}\right)$ and $\left(G, s_{2}\right)$ are switching equivalent, we write $\left(G, s_{1}\right) \sim\left(G, s_{2}\right)$. Figure 1 gives an example of switching equivalent 2-edge-colored graphs where the surrounded vertices belong to $X$. We use the notation $(G)$ for a 2-edge-colored graph when its signature is not relevant or is clear from the context, whereas $G$ refers to the underlying simple graph of $(G)$.

Given two graphs $(G, s)$ and $(H, t), \varphi$ is a 2-edge-colored homomorphism of $(G, s)$ to $(H, t)$ if $\varphi: V(G) \rightarrow V(H)$ is a mapping such that every edge of $(G, s)$ is mapped to an edge of the same sign in $(H, t)$. Given two graphs $\left(G, s_{1}\right)$ and $\left(H, t_{1}\right)$, we say that there is a switching homomorphism $\varphi$ of $\left(G, s_{1}\right)$ to $\left(H, t_{1}\right)$ if there exist $\left(G, s_{2}\right) \sim\left(G, s_{1}\right)$ and $\left(H, t_{2}\right) \sim\left(H, t_{1}\right)$ such that $\varphi$ is a 2-edge-colored homomorphism of $\left(G, s_{2}\right)$ to $\left(H, t_{2}\right)$.

Lemma 1 If $(G, s)$ admits a switching homomorphism to $(H, t)$, then there exists $\left(G, s^{\prime}\right) \sim(G, s)$ such that $\left(G, s^{\prime}\right)$ admits a 2-edge-colored homomorphism to $(H, t)$. 
Proof. Since $(G, s)$ admits a switching homomorphism to $(H, t)$, this implies that there exist $\left(G, s^{\prime \prime}\right) \sim(G, s),\left(H, t^{\prime}\right) \sim(H, t)$, and a 2-edge-colored homomorphism $\varphi$ of $\left(G, s^{\prime \prime}\right)$ to $\left(H, t^{\prime}\right)$. Let $X \subseteq V(H)$ be such that $\left(H, t^{\prime}\right)=$ $\left(H, t^{(X)}\right)$. Let $Y=\{v \in V(G) \mid \varphi(v) \in X\}$. Let $\left(G, s^{\prime}\right)=\left(G, s^{\prime \prime(Y)}\right)$. For every $u v \in E(G)$ we have:

- $s^{\prime}(u v)=-s^{\prime \prime}(u v) \Longleftrightarrow u v \in \partial(Y)$ by the switching of $Y$.

- $t(\varphi(u) \varphi(v))=-t^{\prime}(\varphi(u) \varphi(v)) \Longleftrightarrow \varphi(u) \varphi(v) \in \partial(X)$ by the switching of $X$.

- $u v \in \partial(Y) \Longleftrightarrow \varphi(u) \varphi(v) \in \partial(X)$ by the definition of $Y$.

- $s^{\prime \prime}(u v)=t^{\prime}(\varphi(u) \varphi(v))$ by the definition of $\varphi$.

Then we deduce that $s^{\prime}(u v)=t(\varphi(u) \varphi(v))$, which means that $\varphi$ is a 2-edgecolored homomorphism of $\left(G, s^{\prime}\right)$ to $(H, t)$.

The above lemma implies that, in order to prove the existence of a switching homomorphism of $(G, s)$ to $(H, t)$, there is no need to switch $(H, t)$.

The 2-edge-colored chromatic number $\chi_{2}(G, s)$ of the graph $(G, s)$ is the minimum order (number of vertices) of a graph $(H, t)$ such that $(G, s)$ admits a 2-edge-colored homomorphism to $(H, t)$. Similarly, we define $\chi_{s w}(G, s)$ as the minimum order of a graph $(H, t)$ such that $(G, s)$ admits a switching homomorphism to $(H, t)$. Equivalently, $\chi_{s w}(G, s)=\min \left\{\chi_{2}\left(G, s^{\prime}\right) \mid\left(G, s^{\prime}\right) \sim(G, s)\right\}$.

The 2-edge-colored chromatic number $\chi_{2}(G)$ of a simple graph $G$ is defined as the maximum $\chi_{2}(G)=\max \left\{\chi_{2}(G, s)\right\}$ over all signatures. The 2-edgecolored chromatic number $\chi_{2}(\mathcal{C})$ of a class of simple graphs $\mathcal{C}$ is defined as $\chi_{2}(\mathcal{C})=\max \left\{\chi_{2}(G) \mid G \in \mathcal{C}\right\}$. Similarly, $\chi_{s w}(G)=\max \left\{\chi_{s w}(G, s)\right\}$ and $\chi_{s w}(\mathcal{C})=\max \left\{\chi_{s w}(G) \mid G \in \mathcal{C}\right\}$.

An equivalent definition of the 2-edge-colored chromatic number can be given by defining 2-edge-colored coloring. A 2-edge-colored $k$-coloring of a 2-edgecolored graph $(G, s)$ is a proper vertex-coloring $\varphi: V(G) \rightarrow\{1,2, \ldots, k\}$ such that if there exist two edges $u v$ and $x y$ with $\varphi(u)=\varphi(x)$ and $\varphi(v)=\varphi(y)$, then these two edges have the same sign. Notice that given such a coloring, we can construct the target graph $(H, t)$ associated to $\varphi$ such that $V(H)=$ $\{1,2, \ldots, k\}$ and for every $u v \in E(G)$ there exists $e=\varphi(u) \varphi(v) \in E(H)$ such that $t(e)=s(u v)$. Hence, the 2-edge-colored chromatic number of $(G)$ is the minimum number of colors needed for a 2-edge-colored coloring of $(G)$.

We use the following notations. The set of positive (resp. negative) neighbors of a vertex $v$ in a 2-edge-colored graph is denoted by $N^{+}(v)\left(\right.$ resp. $\left.N^{-}(v)\right)$. A vertex of degree $k$ (resp. at least $k$, at most $k$ ) is called a $k$-vertex (resp. $\geqslant k$-vertex, $\leqslant k$-vertex). A path (resp. a cycle) containing $k$ edges is called a $k$-path (resp. a $k$-cycle).

In this paper, we study 2-edge-colored and switching homomorphisms of outerplanar and planar graphs of given girth. In Section 2, we introduce and study the properties of several families of target graphs, namely the antitwinned graph $A T(G, s)$, the 2-edge-colored Zielonka graph $S Z_{k}$, the 2-edgecolored Paley graph $S P_{q}$, and the 2-edge-colored Tromp Paley graph $\operatorname{Tr}\left(S P_{q}\right)$. 
We consider 2-edge-colored homomorphisms of planar graphs and outerplanar graphs in Section 3 and we provide lower and upper bounds on the 2-edgecolored chromatic number. In particular, we prove that $12 \leqslant \chi_{2}\left(\mathcal{P}_{4}\right) \leqslant 50$. This improves the previous known upper bound of 80 that holds for planar graphs. We obtain results on switching homomorphisms of planar graphs and outerplanar graphs of given girth in Section 4. We finally conclude in Section 5.

\section{Target graphs}

Given a class of simple graphs $\mathcal{C}$, we say that a 2-edge-colored graph $(H)$ is $\mathcal{C}$-universal if every 2-edge-colored graph $(G)$ such that $G \in \mathcal{C}$ admits a 2-edge-colored homomorphism to $(H)$.

In this section, our goal is not only to find target graphs that will give the required upper bounds of our results in Sections 3 and 4. We describe several families of target graphs that may be $\mathcal{C}$-universal, for some classes $\mathcal{C}$, and we determine their properties. We consider below anti-twinned graphs, the 2-edge-colored Zielonka graph $S Z_{k}$, the 2-edge-colored Paley graph and the 2-edge-colored Tromp Paley graph.

We say that a 2-edge-colored graph $(G, s)$ is

- vertex-transitive if for every two vertices $u$ and $v$, there exists a 2-edgecolored automorphism mapping $u$ to $v$.

- arc-transitive if for every vertices $u_{1}, u_{2}, v_{1}$, and $v_{2}$ such that $u_{1} u_{2}$ and $v_{1} v_{2}$ are edges of the same sign, there exists a 2-edge-colored automorphism mapping $u_{1}$ to $v_{1}$ and $u_{2}$ to $v_{2}$.

- triangle-transitive if for every vertices $u_{1}, u_{2}, u_{3}, v_{1}, v_{2}$, and $v_{3}$ such that $u_{1} u_{2} u_{3}$ and $v_{1} v_{2} v_{3}$ are triangles satisfying $s\left(u_{1} u_{2}\right)=s\left(v_{1} v_{2}\right), s\left(u_{2} u_{3}\right)=$ $s\left(v_{2} v_{3}\right)$, and $s\left(u_{3} u_{1}\right)=s\left(v_{3} v_{1}\right)$, there exists a 2-edge-colored automorphism mapping $u_{1}$ to $v_{1}, u_{2}$ to $v_{2}$, and $u_{3}$ to $v_{3}$.

\subsection{Anti-twinned graphs}

In a 2-edge-colored graph, two distinct vertices $u$ and $v$ are twins if $N^{+}(u)=$ $N^{+}(v)$ and $N^{-}(u)=N^{-}(v)$. Also, $u$ and $v$ are anti-twins if $N^{+}(u)=N^{-}(v)$ and $N^{-}(u)=N^{+}(v)$. Note that twins (resp. anti-twins) are necessarily nonadjacent. Moreover, if $u$ has two anti-twins $v_{1}$ and $v_{2}$, then $v_{1}$ and $v_{2}$ are twins. A 2-edge-colored graph is twin-free if it contains neither a pair of twins nor a pair of anti-twins.

Let $(G, s)$ be a 2-edge-colored graph and let $\left(G^{+1}\right)$ and $\left(G^{-1}\right)$ be two copies of $(G)$. The vertex corresponding to $u \in V(G)$ in $\left(G^{i}\right)$ is denoted by $u_{i}$. We define the graph $A T(G, s)=(H, t)$ on $2|V(G)|$ vertices as follows:

- $V(H)=V\left(G^{+1}\right) \cup V\left(G^{-1}\right)$

- $E(H)=\left\{u_{i} v_{j}: u v \in E(G), i \in\{-1,+1\}, j \in\{-1,+1\}\right\}$

- $t\left(u_{i} v_{j}\right)=i \times j \times s(u v)$

Figure 2 illustrates the construction of the graph $A T(G, s)$. 


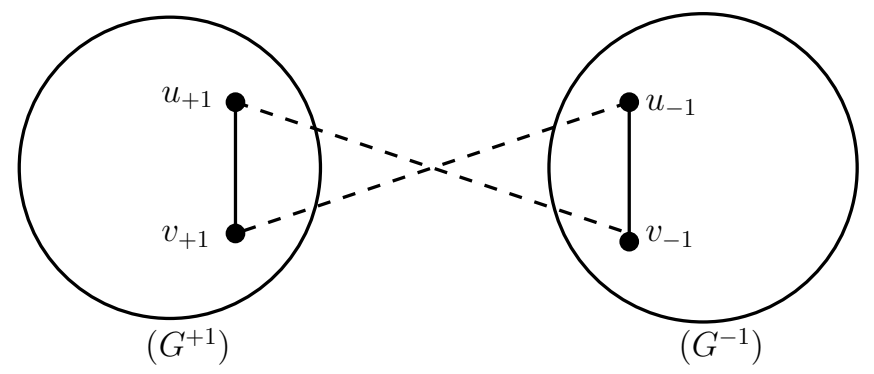

Fig. 2. The graph $A T(G, s)$.

We say that a 2-edge-colored graph $H$ is anti-twinned if and only if every vertex has a unique anti-twin.

Observation 2 A 2-edge-colored graph $H$ is anti-twinned if and only if $H$ is isomorphic to $A T(G, s)$ such that $(G, s)$ is a twin-free 2-edge-colored graph.

Given a twin-free 2-edge-colored graph $(G, s)$, we denote by atw $: V(A T(G, s)) \rightarrow$ $V(A T(G, s))$ the fixed-point-free involution which maps every vertex $u_{i}$ to its unique anti-twin, that is atw $\left(u_{i}\right)=u_{-i}$. Note that atw is an automorphism of $A T(G, s)$. By extension, if $W=\{u, v, \ldots\}$ is a subset of $V(A T(G, s))$, then $\operatorname{atw}(W)=\{\operatorname{atw}(u), \operatorname{atw}(v), \ldots\}$.

Lemma 3 A graph $(G, s)$ admits a switching homomorphism to a twin-free graph $(H, t)$ if and only if $(G, s)$ admits a 2-edge-colored homomorphism to the anti-twinned graph $A T(H, t)$.

Proof. Brewster and Graves [4, Theorem 12] obtained a general result on $m$ edge-colored graphs which gives the following in the case $m=2$ : a graph $(G, s)$ admits a switching homomorphism to a graph $(H, t)$ if and only if $A T(G, s)$ admits a 2-edge-colored homomorphism to $A T(H, t)$.

Notice that $A T(G, s)$ admits a 2-edge-colored homomorphism to $A T(H, t)$ if and only if $(G, s)$ admits a 2-edge-colored homomorphism to $A T(H, t)$. This gives that $(G, s)$ admits a switching homomorphism to a graph $(H, t)$ if and only if $(G, s)$ admits a 2-edge-colored homomorphism to $A T(H, t)$.

Finally, we can conclude by means of Observation 2 .

Corollary 4 If $(G, s)$ admits a 2-edge-colored homomorphism to an antitwinned graph $T$, then $\left(G, s^{\prime}\right)$ admits a 2-edge-colored homomorphism to $T$ for every $\left(G, s^{\prime}\right) \sim(G, s)$.

\subsection{The 2-edge-colored Zielonka graph $S Z_{k}$}

The Zielonka graph $Z_{k}$ is an oriented graph introduced by Zielonka [15] in the theory of bounded timestamp systems. Raspaud and Sopena [12] have used $Z_{k}$ in the context of oriented homomorphism. Alon and Marshall [1] have adapted this construction to $m$-edge-colored graphs in order to get bounds on the $m$-edge-colored chromatic number of graphs having an acyclic $k$-coloring. 


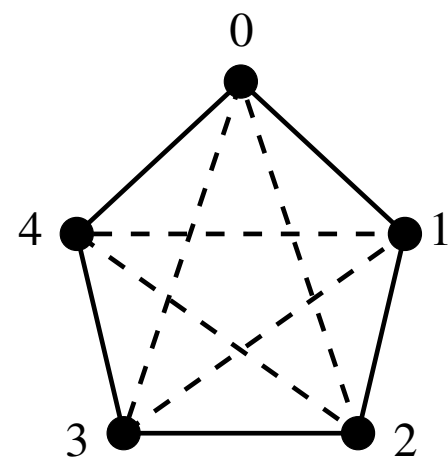

Fig. 3. The 2-edge-colored graph $S P_{5}$.

Let us describe the construction of the 2-edge-colored Zielonka graph $S Z_{k}$ corresponding to the case $m=2$. Every vertex is of the form $\left(i ; \alpha_{1}, \alpha_{2}, \ldots, \alpha_{k}\right)$ where $1 \leqslant i \leqslant k, \alpha_{j} \in\{-1,+1\}$ for $j \neq i$, and $\alpha_{i}=0$. Thus, $\left|V\left(S Z_{k}\right)\right|=$ $k \cdot 2^{k-1}$. For $i \neq j$, there is an edge between the vertices $\left(i ; \alpha_{1}, \alpha_{2}, \ldots, \alpha_{k}\right)$ and $\left(j ; \beta_{1}, \beta_{2}, \ldots, \beta_{k}\right)$ and the sign of this edge is the product $\alpha_{j} \times \beta_{i}$.

Proposition 5 The graph $S Z_{k}$ is anti-twinned.

Proof. We denote by $s$ the signature of $S Z_{k}$. By definition of an anti-twinned graph, we have to show that every vertex of $S Z_{k}$ has a unique anti-twin. To prove that the anti-twin of the vertex $u=\left(i ; \alpha_{1}, \alpha_{2}, \ldots, \alpha_{k}\right)$ is the vertex $u^{\prime}=\left(i ;-\alpha_{1},-\alpha_{2}, \ldots,-\alpha_{k}\right)$, we check that for every edge $u v$, the edge $u^{\prime} v$ exists and that $s\left(u^{\prime} v\right)=-s(u v)$. If $u v$ is an edge, then $v=\left(j ; \beta_{1}, \beta_{2}, \ldots, \beta_{k}\right)$ for some $j \neq i$ and thus $s\left(u^{\prime} v\right)=\left(-\alpha_{j}\right) \times \beta_{i}=-\left(\alpha_{j} \times \beta_{i}\right)=-s(u v)$.

\subsection{The 2-edge-colored Paley graph $S P_{q}$}

In the remainder of this section, $q$ is any prime power such that $q \equiv 1(\bmod 4)$. We denote by $\mathbb{F}_{q}$ the unique (up to isomorphism) finite field of order $q$. Let $g$ be a generator of the multiplicative group $\mathbb{F}_{q}^{*}$ and let $\mathrm{sq}: \mathbb{F}_{q}^{*} \rightarrow\{-1,+1\}$ be the function square defined as $\operatorname{sq}(v)=+1$ if and only if $v$ is a square of $\mathbb{F}_{q}$. Note that $g$ is necessarily a non-square, so that

$$
\operatorname{sq}\left(g^{t}\right)=(-1)^{t}
$$

The Paley graph $P_{q}$ is the undirected graph with vertex set $V\left(P_{q}\right)=\mathbb{F}_{q}$ and edge set $E\left(P_{q}\right)=\{x y \mid \operatorname{sq}(y-x)=+1\}$. Since -1 is a square in $\mathbb{F}_{q}, \operatorname{sq}(x-y)=$ $\mathrm{sq}(y-x)$ and therefore the definition of an edge is consistent. A Paley graph is vertex-transitive, arc-transitive, and self-complementary [13], that is, it is isomorphic to its complement.

A strongly regular graph with parameters $(n, k, \lambda, \mu)$ is a $k$-regular graph $G$ with $n$ vertices such that (1) every two adjacent vertices have $\lambda$ common neighbors and (2) every two non-adjacent vertices have $\mu$ common neighbors. Paley graphs $P_{q}$ are known to be strongly regular graphs with parameters $\left(q, \frac{q-1}{2}, \frac{q-5}{4}, \frac{q-1}{4}\right)$. 


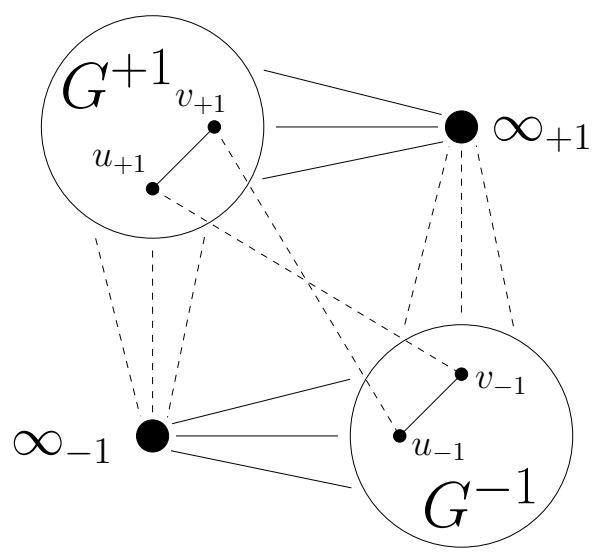

Fig. 4. The 2-edge-colored graph $\operatorname{Tr}(G)$.

We define the 2-edge-colored Paley graph $S P_{q}=\left(K_{q}, s\right)$ as the complete graph on $q$ vertices such that $V\left(S P_{q}\right)=\mathbb{F}_{q}$ and $s(u v)=\mathrm{sq}(u-v)$. That is, $S P_{q}$ is obtained from the Paley graph $P_{q}$ by replacing non-edges by negative edges. Figure 3 represents the 2-edge-colored Paley graph $S P_{5}$.

An anti-automorphism of a 2-edge-colored graph $(G, s)$ is a permutation $\rho$ of $V(G)$ such that for every edge $u v, \rho(u) \rho(v)$ is an edge and $s(\rho(u) \rho(v))=$ $-s(u v)$. Then $(G)$ is anti-automorphic if it admits an anti-automorphism.

Since $P_{q}$ is vertex-transitive, arc-transitive, and self-complementary, $S P_{q}$ is vertex-transitive, arc-transitive, and anti-automorphic.

\subsection{The 2-edge-colored Tromp Paley graph $\operatorname{Tr}\left(S P_{q}\right)$}

Given an oriented graph $\vec{G}$, Tromp [14] proposed a construction of an oriented graph $\operatorname{Tr}(G)$ called the Tromp graph. We adapt this construction to 2-edgecolored graphs as follows.

Given a 2-edge-colored graph $(G)$, we denote by $\left(G^{\bullet}\right)$ the graph obtained from $(G)$ by adding a universal vertex, denoted by $\infty$, that is positively linked to all the vertices of $(G)$.

The Tromp 2-edge-colored graph $\operatorname{Tr}(G)$ of $(G)$ is then defined as the 2-edgecolored graph $A T\left(G^{\bullet}\right)$. By construction, $\operatorname{Tr}(G)$ is obtained from two copies $\left(G^{+1}\right)$ and $\left(G^{-1}\right)$ of $(G)$ and the vertices $\infty_{+1}$ and $\infty_{-1}$ (see Figure 4 ).

Given a 2-edge-colored graph $(G, s)$, let $\operatorname{Tr}(G)$ be the Tromp graph of $(G)$ with signature $s^{\prime}$. Let $u_{i} v_{j} \in \operatorname{Tr}(G)$ such that $u \neq \infty$. If $v \neq \infty$, then

$$
s^{\prime}\left(u_{i} v_{j}\right)=i \times j \times s(u v) .
$$

Otherwise

$$
s^{\prime}\left(u_{i} \infty_{j}\right)=i \times j
$$

Lemma 6 If $(G)$ is anti-automorphic, then $A T(G)$ and $\operatorname{Tr}(G)$ are anti-automorphic. 
Proof. Let $s$ be the signature of $G$ and $s^{\prime}$ be the signature of $\operatorname{Tr}(G)$. Let $\rho$ be an anti-automorphism of $(G)$. We define the mapping $\gamma: \operatorname{Vir}(G)) \rightarrow$ $V(\operatorname{Tr}(G))$ as:

$$
\gamma: u_{i} \rightarrow \begin{cases}\infty_{-i} & \text { if } u=\infty \\ (\rho(u))_{i} & \text { if } u \neq \infty\end{cases}
$$

Let us check that $\gamma$ maps every edge $u_{i} v_{j} \in E(\operatorname{Tr}(G))$ to an edge of opposite sign. If $u, v \neq \infty$, then $\gamma$ maps $u_{i} v_{j}$ to $\rho(u)_{i} \rho(v)_{j}$ and we have by (2):

$$
s^{\prime}\left(\rho(u)_{i} \rho(v)_{j}\right)=i \times j \times s(\rho(u) \rho(v))=i \times j \times(-s(u v))=-s^{\prime}\left(u_{i} v_{j}\right) .
$$

If $u \neq \infty$ and $v=\infty$, then $\gamma$ maps $u_{i} \infty_{j}$ to $\rho(u)_{i} \infty_{-j}$ and we have by (3):

$$
s^{\prime}\left(\rho(u)_{i} \infty_{-j}\right)=i \times(-j)=-(i \times j)=-s^{\prime}\left(u_{i} \infty_{j}\right) .
$$

Since the restriction of $\gamma$ to $V(A T(G))$ is well-defined, the same proof applies to $A T(G)$.

In the remainder of this section, we focus on the Tromp Paley graph $\operatorname{Tr}\left(S P_{q}\right)$ obtained by applying the Tromp construction to the 2-edge-colored Paley graph $S P_{q}$. It has $2 q+2$ vertices denoted $u_{i}$ such that $u \in\left\{0,1=g^{0}, g, g^{2}, \ldots\right.$, $\left.g^{q-2}, \infty\right\}$ and $i \in\{-1,+1\}$ (recall that $g$ is a generator of the multiplicative group $\left.\mathbb{F}_{q}^{*}\right)$. We denote by $s$ the signature of $S P_{q}$ and by $s^{\prime}$ the signature of $\operatorname{Tr}\left(S P_{q}\right)$. Note that if $u_{i} v_{j} \in \operatorname{Tr}\left(S P_{q}\right), u \neq \infty$, and $v \neq \infty$, then $s^{\prime}\left(u_{i} v_{j}\right)=i \times j \times \mathrm{sq}(u-v)$ by $(2)$ since $s(u v)=\mathrm{sq}(u-v)$.

The graph $\operatorname{Tr}\left(S P_{q}\right)$ has remarkable symmetries and some useful properties given below.

Lemma 7 The 2-edge-colored graph $\operatorname{Tr}\left(S P_{q}\right)$ is vertex-transitive.

Proof. To prove that $\operatorname{Tr}\left(S P_{q}\right)$ is vertex-transitive, we show that every vertex $u$ can be mapped to $\infty_{+1}$. Recall that $S P_{q}$ is vertex-transitive and arctransitive. Moreover, for every vertex $w_{i} \in V\left(\operatorname{Tr}\left(S P_{q}\right)\right)$, either $w=\infty, w=0$, or $w=g^{t}$ for some $t$.

If $\varphi$ is an automorphism of $S P_{q}$, we define the corresponding automorphism $\gamma_{\varphi}$ of $\operatorname{Tr}\left(S P_{q}\right)$ as:

$$
\gamma_{\varphi}: u_{i} \rightarrow \begin{cases}u_{i} & \text { if } u=\infty \\ (\varphi(u))_{i} & \text { if } u \neq \infty\end{cases}
$$

We also define the mapping $\gamma_{\infty}: V\left(\operatorname{Tr}\left(S P_{q}\right)\right) \rightarrow V\left(\operatorname{Tr}\left(S P_{q}\right)\right)$ as:

$$
\gamma_{\infty}: u_{i} \rightarrow \begin{cases}\infty_{i} & \text { if } u=0 \\ 0_{i} & \text { if } u=\infty \\ g_{i \times(-1)^{t}}^{-t} & \text { if } u=g^{t}\end{cases}
$$


Let us check that $\gamma_{\infty}$ is an automorphism of $\operatorname{Tr}\left(S P_{q}\right)$, i.e., that for every edge $u_{i} v_{j}$, we have $\gamma_{\infty}\left(u_{i}\right) \gamma_{\infty}\left(v_{j}\right) \in E\left(\operatorname{Tr}\left(S P_{q}\right)\right)$ and $s^{\prime}\left(\gamma_{\infty}\left(u_{i}\right) \gamma_{\infty}\left(v_{j}\right)\right)=s^{\prime}\left(u_{i} v_{j}\right)$.

- If $u=g^{t}$ and $v=g^{r}$, we have

$$
\begin{aligned}
s^{\prime}\left(\gamma_{\infty}\left(u_{i}\right) \gamma_{\infty}\left(v_{j}\right)\right) & =s^{\prime}\left(g_{i \times(-1)^{t}}^{-t} g_{j \times(-1)^{r}}^{-r}\right) \\
& =i \times(-1)^{t} \times j \times(-1)^{r} \times \mathrm{sq}\left(g^{-t}-g^{-r}\right) \\
& =i \times j \times \mathrm{sq}\left(g^{t+r}\right) \times \mathrm{sq}\left(g^{-t}-g^{-r}\right) \\
& =i \times j \times \mathrm{sq}\left(g^{t+r}\left(g^{-t}-g^{-r}\right)\right) \\
& =i \times j \times \mathrm{sq}\left(g^{r}-g^{t}\right) \\
& =i \times j \times \mathrm{sq}\left(g^{t}-g^{r}\right) \\
& =s^{\prime}\left(g_{i}^{t} g_{j}^{r}\right) \\
& =s^{\prime}\left(u_{i} v_{j}\right) .
\end{aligned}
$$

- If $u=g^{t}$ and $v=0$, we have

$$
\begin{aligned}
s^{\prime}\left(\gamma_{\infty}\left(u_{i}\right) \gamma_{\infty}\left(v_{j}\right)\right) & =s^{\prime}\left(g_{i \times(-1)^{t}}^{-t} \infty_{j}\right) \\
& =i \times(-1)^{t} \times j \\
& =i \times j \times \operatorname{sq}\left(g^{t}\right) \\
& =i \times j \times \operatorname{sq}\left(g^{t}-0\right) \\
& =s^{\prime}\left(g_{i}^{t} 0_{j}\right) \\
& =s^{\prime}\left(u_{i} v_{j}\right) .
\end{aligned}
$$

- If $u=g^{t}$ and $v=\infty$, we have

$$
\begin{aligned}
s^{\prime}\left(\gamma_{\infty}\left(u_{i}\right) \gamma_{\infty}\left(v_{j}\right)\right) & =s^{\prime}\left(g_{i \times(-1)^{t}}^{-t} 0_{j}\right) \\
& =i \times(-1)^{t} \times j \times \mathrm{sq}\left(g^{-t}-0\right) \\
& =i \times(-1)^{t} \times j \times(-1)^{-t} \\
& =i \times j \\
& =s^{\prime}\left(g_{i}^{t} \infty_{j}\right) \\
& =s^{\prime}\left(u_{i} v_{j}\right) .
\end{aligned}
$$

- If $u=0$ and $v=\infty$, we have

$$
\begin{array}{rlrl}
s^{\prime}\left(\gamma_{\infty}\left(u_{i}\right) \gamma_{\infty}\left(v_{j}\right)\right) & =s^{\prime}\left(\infty_{i} 0_{j}\right) & \\
& =i \times j & & \text { by }(3) \\
& =s^{\prime}\left(0_{i} \infty_{j}\right) & & \text { by }(3) \\
& =s^{\prime}\left(u_{i} v_{j}\right) . & &
\end{array}
$$


Given a vertex $u \in S P_{q}$, the automorphism $\varphi$ can be chosen so that $\varphi(u)=$ 0 . Then, we have $\gamma_{\varphi}\left(u_{i}\right)=0_{i}$ for every $u \neq \infty$. Also, $\gamma_{\infty}\left(0_{i}\right)=\infty_{i}$ and $\operatorname{atw}\left(\infty_{-1}\right)=\infty_{+1}$. Thus, we can map every vertex of $\operatorname{Tr}\left(S P_{q}\right)$ to $\infty_{+1}$ by combining the automorphisms $\gamma_{\varphi}, \gamma_{\infty}$, and atw. So $\operatorname{Tr}\left(S P_{q}\right)$ is vertex-transitive.

Lemma $8 \operatorname{Tr}\left(S P_{q}\right)$ is triangle-transitive.

Proof. There are 4 types of triangles $(a, b, c)$ according to the number of positive edges in their signature. Without loss of generality, the edges incident to $c$ have the same sign $\beta$ and we denote by $\alpha$ the sign of $a b$. Thus, every type of triangle is characterized by a couple $(\alpha, \beta) \in\{-1,+1\}^{2}$. We have to prove that for every two triangles of the same type, there exists an automorphism of $\operatorname{Tr}\left(S P_{q}\right)$ that maps one triangle to the other.

We first prove that every triangle $(a, b, c)$ of type $(+1, \beta)$ maps to the triangle $0_{+1} 1_{+1} \infty_{\beta}$. By Lemma $7, \operatorname{Tr}\left(S P_{q}\right)$ is vertex-transitive, so there exists an automorphism $\varphi$ that maps $c$ to $\infty_{\beta}$. Every edge of $\operatorname{sign} \beta$ incident to $\infty_{\beta}$ has its other extremity in $S P_{q}^{+1}$. Thus, $\varphi$ maps the edge $a b$ to a positive edge $u_{+1} v_{+1}$ in $S P_{q}^{+1}$. Since $S P_{q}$ is arc-transitive, we can finally map $u_{+1} v_{+1}$ to $0_{+1} 1_{+1}$.

Let $\overline{\operatorname{Tr}\left(S P_{q}\right)}$ be obtained from $\operatorname{Tr}\left(S P_{q}\right)$ by changing the sign of every edge. Since $S P_{q}$ is anti-automorphic, $\operatorname{Tr}\left(S P_{q}\right)$ is also anti-automorphic by Lemma 6 . So $\overline{\operatorname{Tr}\left(S P_{q}\right)}$ is isomorphic to $\operatorname{Tr}\left(S P_{q}\right)$. Let us fix $\beta \in\{-1,+1\}$ and let $T_{1}$ and $T_{2}$ be two triangles of type $(-1, \beta)$ in $\operatorname{Tr}\left(S P_{q}\right)$. So $T_{1}$ and $T_{2}$ are triangles of type $(+1,-\beta)$ in $\overline{\operatorname{Tr}\left(S P_{q}\right)}$. By the previous case, there exists an automorphism that maps $T_{1}$ to $T_{2}$ in $\overline{\operatorname{Tr}\left(S P_{q}\right)}$. This automorphism also maps $T_{1}$ to $T_{2}$ in $\operatorname{Tr}\left(S P_{q}\right)$.

\subsection{Coloring properties of target graphs}

A sign vector of size $k$ is a $k$-tuple $\alpha=\left(\alpha_{1}, \alpha_{2}, \ldots, \alpha_{k}\right) \in\{-1,+1\}^{k}$. The negation of $\alpha$ is the sign vector $-\alpha=\left(-\alpha_{1},-\alpha_{2}, \ldots,-\alpha_{k}\right)$. Given a sequence of $k$ distinct vertices $X_{k}=\left(v_{1}, v_{2}, \ldots, v_{k}\right)$ of a 2-edge-colored graph $(G, s)$ that induces a clique in $G$, an $\alpha$-neighbor of $X_{k}$ is a vertex $u \in V(G)$ such that $s\left(v_{i} u\right)=\alpha_{i}$ for $1 \leqslant i \leqslant k$. The set of $\alpha$-neighbors of $X_{k}$ is denoted by $N^{\alpha}\left(X_{k}\right)$. Thus, the notion of $\alpha$-neighbor generalizes to sequences of vertices the notions of positive and negative neighbors of a vertex.

Consider the 2-edge-colored graph $S P_{5}$ depicted in Figure 3. For example, given $\alpha=(+1,-1)$ and $X=(0,3)$, the vertex 1 is an $\alpha$-neighbor of $X$, the vertex 2 is a $(-\alpha)$-neighbor of $X$, we have $N^{\alpha}(X)=\{1\}$ and $N^{-\alpha}(X)=\{2\}$.

A 2-edge-colored graph $(G)$ has property $P_{k, l}$ if $\left|N^{\alpha}\left(X_{k}\right)\right| \geqslant l$ for every sequence $X_{k}$ of $k$ distinct vertices inducing a clique in $G$ and for every sign vector $\alpha$ of size $k$.

Lemma 9 If $S P_{q}$ has property $P_{n-1, k}$, then $\operatorname{Tr}\left(S P_{q}\right)$ has property $P_{n, k}$.

Proof. Suppose that $S P_{q}$ has property $P_{n-1, k}$ and let $\alpha=\left(\alpha_{1}, \ldots, \alpha_{n-1}, \alpha_{n}\right)$ be a given sign vector. Let $X=\left(x_{1}, \ldots, x_{n-1}, x_{n}\right)$ be a sequence of $n$ distinct 
vertices inducing a clique of $\operatorname{Tr}\left(S P_{q}\right)$. We have to prove that $X$ admits $k \alpha$ neighbors. By Lemma $7, \operatorname{Tr}\left(S P_{q}\right)$ is vertex-transitive and we assume without loss of generality that $x_{n}=\infty_{+1}$. By noticing that $N^{-\alpha}(X)=\operatorname{atw}\left(N^{\alpha}(X)\right)$, we also assume without loss of generality that $\alpha_{n}=+1$. Since $X$ is a clique, we have $x_{i} \notin\left\{\infty_{+1}, \infty_{-1}\right\}$ for $1 \leqslant i \leqslant n-1$. We define $Y=\left(y_{1}, \ldots, y_{n-1}\right)$ such that $y_{i}=x_{i}$ if $x_{i} \in S P_{q}^{+1}$ and $y_{i}=\operatorname{atw}\left(x_{i}\right)$ if $x_{i} \in S P_{q}^{-1}$. Hence, the vertices in $Y$ belong to $S P_{q}^{+1}$. We define $\beta=\left(\beta_{1}, \ldots, \beta_{n-1}\right)$ such that, for $1 \leqslant i \leqslant n-1, \beta_{i}=\alpha_{i}$ if and only if $x_{i}=y_{i}$. By Property $P_{n-1, k}$ of $S P_{q}$, there exist $k \beta$-neighbors $v_{1}, v_{2}, \ldots, v_{k}$ of $Y$ in $S P_{q}^{+1}$. The $v_{i}$ 's are positive neighbors of $\infty_{+1}$, so they are $\left(\beta_{1}, \ldots, \beta_{n-1},+1\right)$-neighbors of $\left(y_{1}, \ldots, y_{n-1}, \infty_{+1}\right)$. Hence $X$ has $k \alpha$-neighbors.

Lemma 10 If $(G)$ is a 2-edge-colored graph and $\operatorname{Tr}(G)$ has property $P_{n, k}$, then $A T(G)$ has property $P_{n, k-1}$.

Proof. Recall that $A T(G)$ is obtained from two isomorphic copies of $(G)$ and then $\operatorname{Tr}(G)$ is obtained from $A T(G)$ by adding the anti-twin vertices $\infty_{+1}$ and $\infty_{-1}$. Let $X$ be a sequence of $n$ distinct vertices inducing a clique in $A T(G)$. Since $\operatorname{Tr}(G)$ has property $P_{n, k}$, we have that $N^{\alpha}(X)$ contains $k$ vertices of $\operatorname{Tr}(G)$ for every sign vector $\alpha$ of length $n$. However, $N^{\alpha}(X)$ cannot contain both $\infty_{+1}$ and $\infty_{-1}$, since they are anti-twins. So $X$ has at least $k-1$ $\alpha$-neighbors in $A T(G)$, which means that $A T(G)$ has property $P_{n, k-1}$.

\section{Lemma 11}

(1) $S P_{q}$ has properties $P_{1,(q-1) / 2}$ and $P_{2,(q-5) / 4}$.

(2) $\operatorname{Tr}\left(S P_{q}\right)$ has properties $P_{1, q}, P_{2,(q-1) / 2}$, and $P_{3,(q-5) / 4}$.

(3) $A T\left(S P_{q}\right)$ has properties $P_{1, q-1}, P_{2,(q-3) / 2}$, and $P_{3, \max (0,(q-9) / 4)}$.

\section{Proof.}

(1) These properties follow from the fact that the 2-edge-colored Paley graph $S P_{q}$ is built from the Paley graph $P_{q}$ which is self-complementary, arctransitive, and strongly regular with parameters $\left(q, \frac{q-1}{2}, \frac{q-5}{4}, \frac{q-1}{4}\right)$.

(2) $\operatorname{Tr}\left(S P_{q}\right)$ has property $P_{1, q}$ since it is vertex-transitive by Lemma 7 and the vertex $\infty_{+1}$ has $q$ positive and $q$ negative neighbors. The other properties follow from Lemma 11.(1) and Lemma 9.

(3) These properties follow from Lemma 11.(2) and Lemma 10.

\section{Results on 2-edge-colored homomorphisms}

This section is devoted to 2-edge-colored homomorphisms of planar graphs and outerplanar graphs. Recall that a graph $(H)$ is $\mathcal{C}$-universal if every 2 edge-colored graph $(G)$ of the class $\mathcal{C}$ admits a 2-edge-colored homomorphism to $(H)$.

An acyclic $k$-coloring is a proper vertex-coloring such that every cycle has at least three colors. In other words, the graph induced by every two color classes is a forest. Let $\mathcal{A}_{k}$ be the class of graphs that admit a $k$-acyclic coloring. 
In 1998, Alon and Marshall [1] proved the following (the 2-edge-colored graph $S Z_{k}$ has $k \cdot 2^{k-1}$ vertices and has been considered in Section 2.2):

Theorem 12 ([1]) $S Z_{k}$ is $\mathcal{A}_{k}$-universal. Thus $\chi_{2}\left(\mathcal{A}_{k}\right) \leqslant k \cdot 2^{k-1}$.

Huemer et al. [6] have shown that Theorem 12 is tight.

The girth of a graph is the length of a shortest cycle. We denote by $\mathcal{P}_{g}$ (resp. $\mathcal{O}_{g}$ ) the class of planar graphs (resp. outerplanar graphs) with girth at least $g$. So $\mathcal{P}_{3}$ is simply the class of planar graphs.

Borodin [2] proved that every planar graph admits an acyclic 5-coloring. Thus, Alon and Marshall deduced the following from Theorem 12:

Corollary $13([\mathbf{1}]) S Z_{5}$ is $\mathcal{P}_{3}$-universal. Thus $\chi_{2}\left(\mathcal{P}_{3}\right) \leqslant 80$.

In this same context, Borodin et al. [3] and Montejano et al. [8] obtained the following results:

\section{Theorem $14([3,8])$}

(1) $\operatorname{Tr}\left(S P_{9}\right)$ is $\mathcal{P}_{5}$-universal. Thus $\chi_{2}\left(\mathcal{P}_{5}\right) \leqslant 20$ [8].

(2) $\operatorname{Tr}\left(S P_{5}\right)$ is $\mathcal{P}_{6}$-universal. Thus $\chi_{2}\left(\mathcal{P}_{6}\right) \leqslant 12$ [8].

(3) $S P_{9} \backslash\{0\}$ is $\mathcal{P}_{8}$-universal. Thus $\chi_{2}\left(\mathcal{P}_{8}\right) \leqslant 8$ [8].

(4) $S P_{5}$ is $\mathcal{P}_{13}$-universal. Thus $\chi_{2}\left(\mathcal{P}_{13}\right) \leqslant 5$ [3].

In this section, we obtain anti-twinned target graphs for triangle-free outerplanar graphs (Theorem 15) and triangle-free planar graphs (Theorem 16). The latter result gives a new upper bound on the 2-edge-colored chromatic number. Then, we give properties that must be satisfied by the target graphs for outerplanar graphs (Theorem 18) and planar graphs (Theorem 19). Finally, we obtain new lower bounds on the 2-edge-colored chromatic number of triangle-free planar graphs (Theorem 20) and planar graphs with girth at least 7 (Theorem 21).

Theorem $15 A T\left(S P_{5} \backslash\{0\}\right)$ is $\mathcal{O}_{4}$-universal.

Proof. Assume by contradiction that $(H)$ is a counterexample to the result such that $H$ is minimal with respect to the number of vertices.

Suppose that $H$ contains a $\leqslant 1$-vertex $u$. By minimality of $H$, the graph $\left(H^{\prime}\right)=$ $(H \backslash\{u\})$ admits a 2-edge-colored homomorphism to $A T\left(S P_{5} \backslash\{0\}\right)$. Since every vertex of $A T\left(S P_{5} \backslash\{0\}\right)$ is incident to a positive and a negative edge, we can extend the 2-edge-colored homomorphism to $(H)$, a contradiction.

Suppose that $H$ contains two adjacent 2-vertices $u$ and $v$. By minimality of $H$, the graph $\left(H^{\prime}\right)=(H \backslash\{u, v\})$ admits a 2-edge-colored homomorphism to $A T\left(S P_{5} \backslash\{0\}\right)$. We have checked that for every pair of (non necessarily distinct) vertices $x$ and $y$ of $A T\left(S P_{5} \backslash\{0\}\right)$, the 8 possible 2-edge-colored 3paths exist. Therefore, we can extend the 2-edge-colored homomorphism to $(H)$, a contradiction.

Pinlou and Sopena [11] have shown that every outerplanar graph with girth at least $k$ and minimum degree at least 2 contains a face of length $l \geqslant k$ 
with at least $(l-2)$ consecutive 2 -vertices. Therefore, $H$ is not a triangle-free outerplanar graph. This contradiction completes the proof.

Theorem $16 A T\left(S P_{25}\right)$ is $\mathcal{P}_{4}$-universal. Thus $\chi_{2}\left(\mathcal{P}_{4}\right) \leqslant 50$.

Let $n_{3}(G)$ be the number of $\geqslant 3$-vertices in the graph $G$. Let us define the partial order $\preceq$. Given two graphs $G_{1}$ and $G_{2}$, we have $G_{1} \prec G_{2}$ if and only if one of the following conditions holds:

- $n_{3}\left(G_{1}\right)<n_{3}\left(G_{2}\right)$.

- $n_{3}\left(G_{1}\right)=n_{3}\left(G_{2}\right)$ and $\left|V\left(G_{1}\right)\right|+\left|E\left(G_{1}\right)\right|<\left|V\left(G_{2}\right)\right|+\left|E\left(G_{2}\right)\right|$.

Note that the partial order $\preceq$ is well-defined and is a partial linear extension of the minor poset.

Let $(H)$ be a 2-edge-colored graph that does not admit a homomorphism to the 2-edge-colored graph $A T\left(S P_{25}\right)$ and such that its underlying graph $H$ is a triangle-free planar graph which is minimal with respect to $\preceq$. In the following, $H$ is given with its embedding in the plane. A weak 7 -vertex $u$ in $H$ is a 7 vertex adjacent to four 2 -vertices $v_{1}, \ldots, v_{4}$ and three $\geqslant 3$-vertices $w_{1}, w_{2}, w_{3}$ such that $v_{1}, w_{1}, v_{2}, w_{2}, v_{3}, w_{3}$, and $v_{4}$ are clockwise consecutive.

Lemma 17 The graph $H$ does not contain the following configurations:

(C1) $a \leqslant 1$-vertex;

(C2) a k-vertex adjacent to $k 2$-vertices for $2 \leqslant k \leqslant 49$;

(C3) a $k$-vertex adjacent to $(k-1) 2$-vertices for $2 \leqslant k \leqslant 24$;

(C4) a $k$-vertex adjacent to $(k-2) 2$-vertices for $3 \leqslant k \leqslant 12$;

(C5) a 3-vertex;

(C6) a k-vertex adjacent to $(k-3) 2$-vertices for $4 \leqslant k \leqslant 6$;

$(C 7)$ two vertices $u$ and $v$ linked by two distinct 2 -paths, both paths having a 2 -vertex as internal vertex;

(C8) a 4-face wxyz such that $x$ is 2-vertex, $w$ and $y$ are weak 7 -vertices, and $z$ is a $k$-vertex adjacent to $(k-4) 2$-vertices for $4 \leqslant k \leqslant 9$;

Proof. Configurations C2 - C8 are depicted in Figures 5 and 6. The drawing conventions for a configuration $\mathrm{C} k$ contained in a graph $H$ are as follows. The neighbors of a white vertex in $H$ are exactly its neighbors in $\mathrm{C} k$, whereas a black vertex may have other neighbors in $H$. Two or more black vertices in $\mathrm{C} k$ may coincide in a single vertex in $H$, provided they do not share a common white neighbor.

For each configuration, we suppose that $H$ contains the configuration and we consider a 2-edge-colored triangle-free planar graph $\left(H^{\prime}\right)$ such that $H^{\prime} \prec H$. We only argue that $H^{\prime} \prec H$ for configuration C5. For every other configuration, $H^{\prime}$ is a minor of $H$ and thus $H^{\prime} \prec H$. By minimality of $H,\left(H^{\prime}\right)$ admits a 2-edge-colored homomorphism $f$ to $A T\left(S P_{25}\right)$. We modify and extend $f$ to obtain a 2-edge-colored homomorphism of $(H)$ to $A T\left(S P_{25}\right)$, contradicting the fact that $(H)$ is a counterexample.

By Lemma 11.3, $A T\left(S P_{25}\right)$ satisfies $P_{1,24}, P_{2,11}$, and $P_{3,4}$. 
$2 \leq k \leq 49$
$v \underbrace{}_{v_{k}} \quad v_{1}^{\prime}$

(a) $\mathrm{C} 2$

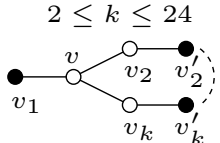

(b) $\mathrm{C} 3$

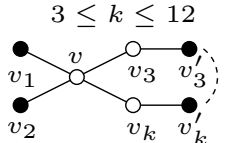

(c) $\mathrm{C} 4$

Fig. 5. Configurations $\mathrm{C} 2-\mathrm{C} 4$.

Proof of configuration C1: Trivial.

Proof of configuration C2: Suppose that $H$ contains the configuration depicted in Figure 5(a) and $f$ is a 2-edge-colored homomorphism of $\left(H^{\prime}\right)=$ $(H) \backslash\left\{v, v_{1}, \ldots, v_{k}\right\}$ to $A T\left(S P_{25}\right)$. For every $i$, if the edges $v v_{i}$ and $v_{i} v_{i}^{\prime}$ have the same sign (resp. different signs), then $v$ must get a color distinct from $\operatorname{atw}\left(f\left(v_{i}^{\prime}\right)\right)$ (resp. $f\left(v_{i}^{\prime}\right)$ ). So, each $v_{i}^{\prime}$ forbids at most one color for $v$. Thus there remains an available color for $v$. Then we extend $f$ to the vertices $v_{i}$ using property $P_{2,11}$ if $f\left(v_{i}^{\prime}\right) \neq f(v)$ of or $P_{1,24}$ if $f\left(v_{i}^{\prime}\right)=f(v)$.

Proof of configuration C3: Suppose that $H$ contains the configuration depicted in Figure 5(b) and $f$ is a 2-edge-colored homomorphism of $\left(H^{\prime}\right)=$ $(H) \backslash\left\{v, v_{2}, \ldots, v_{k}\right\}$ to $A T\left(S P_{25}\right)$. As shown in the proof of Configuration C2, each $v_{i}^{\prime}$ forbids at most one color for $v$. So, we have at most 23 forbidden colors for $v$ and by property $P_{1,24}$, there remains at least one available color for $v$. Then we extend $f$ to the vertices $v_{i}(2 \leqslant i \leqslant k)$ using property $P_{2,11}$.

Proof of configuration $C_{4}$ : Suppose that $H$ contains the configuration depicted in Figure 5(c) and $f$ is a 2-edge-colored homomorphism of $\left(H^{\prime}\right)=$ $(H) \backslash\left\{v_{3}, \ldots, v_{k}\right\}$ to $A T\left(S P_{25}\right)$. As shown in the proof of Configuration C2, each $v_{i}^{\prime}$ forbids at most one color for $v$. So, we have at most 10 forbidden colors for $v$ and by property $P_{2,11}$ applied to $f\left(v_{1}\right), f\left(v_{2}\right)$ this remains at least one available color in order to recolor $v$. Then we extend $f$ to the vertices $v_{i}$ $(3 \leqslant i \leqslant k)$ using property $P_{2,11}$.

Proof of configuration C5: Suppose that $H$ contains the configuration depicted in Figure $6(\mathrm{a})$. Let $\left(H^{\prime}\right)$ be the graph obtained from $(H)$ by deleting the vertex $v$ and by adding, for every $1 \leqslant i<j \leqslant 3$, a new vertex $v_{i j}$ and the edges $v_{i} v_{i j}$ and $v_{i j} v_{j}$. Each of the 6 edges $v_{i} v_{i j}$ gets the sign $\alpha_{i}$ of the edge $v_{i} v$ in $(H)$. Since configuration $\mathrm{C} 4$ is forbidden in $H, v_{1}, v_{2}$ and $v_{3}$ are ${ }^{\geqslant} 3$-vertices. We have $H^{\prime} \prec H$ since $n_{3}\left(H^{\prime}\right)<n_{3}(H)$. Clearly, $H^{\prime}$ is triangle free. Hence, there exists a 2-edge-colored homomorphism $f$ of $\left(H^{\prime}\right)$ to $A T\left(S P_{25}\right)$. By $P_{3,4}$, we can find an $\alpha$-neighbor $u$ of $\left(f\left(v_{1}\right), f\left(v_{2}\right), f\left(v_{3}\right)\right)$ in $A T\left(S P_{25}\right)$ with $\alpha=\left(\alpha_{1}, \alpha_{2}, \alpha_{3}\right)$. Now fix $f(v)=u$. Note that $f$ restricted to $V(H)$ is a homomorphism of $(H)$ to $A T\left(S P_{25}\right)$.

Proof of configuration C6: Suppose that $H$ contains the configuration depicted in Figure 6(b) and $f$ is a 2-edge-colored homomorphism of $\left(H^{\prime}\right)=$ $(H) \backslash\left\{v_{4}, \ldots, v_{k}\right\}$ to $A T\left(S P_{25}\right)$. As shown in the proof of Configuration C2, each $v_{i}^{\prime}$ forbids at most one color for $v$. So, we have at most 3 forbidden colors for $v$ and by property $P_{3,4}$ applied to $f\left(v_{1}\right), f\left(v_{2}\right), f\left(v_{3}\right)$, there remains at least 


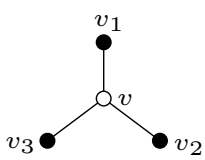

(a) $\mathrm{C} 5$

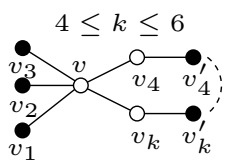

(b) $\mathrm{C} 6$

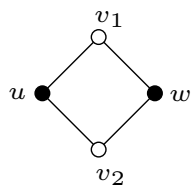

(c) $\mathrm{C} 7$

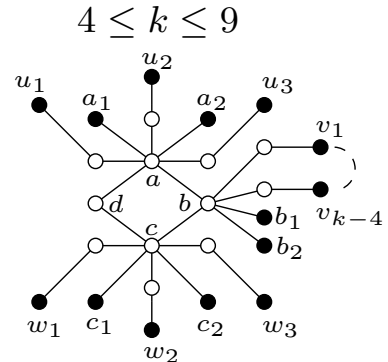

(d) $\mathrm{C} 8$

Fig. 6. Configurations C5-C8.

one available color for $v$. Then we extend $f$ to the vertices $v_{i}(4 \leqslant i \leqslant k)$ using property $P_{2,11}$.

Proof of configuration $\boldsymbol{C}$ \%: Suppose that $H$ contains the configuration depicted in Figure 6(c).

If $u$ and $w$ have no common neighbor other than $v_{1}$ and $v_{2}$, then we consider the graph $\left(H^{\prime}\right)$ obtained from $(H) \backslash\left\{v_{1}, v_{2}\right\}$ by adding the positive edge $u w$.

If $u$ and $w$ have at least one other common neighbor $v_{3}$, then consider the graph $\left(H^{\prime}\right)$ obtained from $(H) \backslash\left\{v_{1}, v_{2}\right\}$ by adding a vertex $v$ adjacent to $u$ and $w$ such that $u v$ is negative and the sign of $v w$ is the product of the signs of $u v_{3}$ and $v_{3} w$. Therefore, we have at least two 2-paths linking $u$ and $w$, one whose both edges have the same sign and one whose edges have different signs.

In both cases, $H^{\prime}$ is triangle-free, planar, and is a minor of $H$, so that $\left(H^{\prime}\right)$ admits a 2-edge-colored homomorphism $f$ to $A T\left(S P_{25}\right)$. Also, in both cases, $f(u)$ and $f(w)$ form an edge in $A T\left(S P_{25}\right)$ since $f(u) \neq f(w)$ and $f(u) \neq$ $f(\operatorname{atw}(w))$. Thus, the coloring of $(H) \backslash\left\{v_{1}, v_{2}\right\}$ induced by $f$ can be extended to $(H)$ using property $P_{2,11}$.

Proof of configuration C8: Suppose that $H$ contains the configuration depicted in Figure 6(d). By Corollary 4, $(H)$ admits a 2-edge-colored homomorphism to $A T\left(S P_{25}\right)$ if and only if every 2-edge colored graph that is switching equivalent to $(H)$ admits a 2-edge-colored homomorphism to $A T\left(S P_{25}\right)$. So, by switching a subset of vertices in $\left\{a, b, c, c_{1}, c_{2}\right\}$, we can assume that the edges $d a, a b, b c, c c_{1}$, and $c c_{2}$ are positive. Consider a 2-edge-colored homomorphism $f$ of $\left(H^{\prime}\right)=(H \backslash\{d\})$ to $A T\left(S P_{25}\right)$. The edge $d c$ in $(H)$ has to be negative, since otherwise $f$ would be extendable to $(H)$ by setting $f(d)=f(b)$. Also, we must have $f(c)=f(a)$, since otherwise we could color $d$ using property $P_{2,11}$. In the remainder of the proof, we show that we can modify $f$ such that $f(c) \neq f(a)$. For $1 \leqslant i \leqslant 3$, let $k_{i}$ denote the color that is forbidden for $c$ by $w_{i}$, that is, $k_{i}=f\left(w_{i}\right)$ if the edges of the 2-path linking $c$ and $w_{i}$ have distinct signs and $k_{i}=\operatorname{atw}\left(f\left(w_{i}\right)\right)$ otherwise.

First, we rule out the cases in which $f\left(c_{1}\right), f\left(c_{2}\right)$, and $f(b)$ have at least 5 common positive neighbors, since this allows to choose for $c$ a color that is 


\begin{tabular}{|c|c|c|}
\hline$\left(0_{+1}, 1_{+1}, 2_{+1}\right)$ & $4_{+1}$ & $(1+2 \sqrt{2})_{-1},(1+3 \sqrt{2})_{-1}$ \\
\hline$\left(0_{+1}, 1_{+1}, 3_{+1}\right)$ & $4_{+1}$ & $(3+\sqrt{2})_{-1}, \quad(3+4 \sqrt{2})_{-1}$ \\
\hline$\left(0_{+1}, 1_{+1}, 4_{+1}\right)$ & $3_{+1}$ & $(2 \sqrt{2})_{-1}$ \\
\hline$\left(0_{+1}, 1_{+1},(3+2 \sqrt{2})_{+1}\right)$ & $(3+\sqrt{2})_{-1},(3 \sqrt{2})$ & $(1+3 \sqrt{2})_{-1},(3+4 \sqrt{2})_{-1}$ \\
\hline$\left(0_{+1}, 1_{+1},(3+3 \sqrt{2})_{+1}\right)$ & $+\sqrt{2})_{-1},(2 \sqrt{2})$ & $(1+2 \sqrt{2})_{-1},(3+$ \\
\hline
\end{tabular}

Tab. 7. Sets of the form $\left(0_{+1}, 1_{+1}, \beta_{+1}\right)$ having exactly $4(+1,+1,+1)$-neighbors in $A T\left(S P_{25}\right)$.

not in $\left\{k_{1}, k_{2}, k_{3}, f(a)\right\}$. If $f\left(c_{1}\right), f\left(c_{2}\right)$, and $f(b)$ are not pairwise distinct, then they have at least 11 common positive neighbors by property $P_{2,11}$. So we assume that $f\left(c_{1}\right), f\left(c_{2}\right)$, and $f(b)$ are distinct. We define the sequence $X=\left(f\left(c_{1}\right), f\left(c_{2}\right), f(b)\right)$ and the sign vector $\alpha=(+1,+1,+1)$. Recall that $A T\left(S P_{25}\right)$ contains two copies $S P_{25}^{+1}$ and $S P_{25}^{-1}$ of $S P_{25}$. We consider the graph $\operatorname{Tr}\left(S P_{25}\right)$ obtained by adding the anti-twin vertices $\infty_{+1}$ and $\infty_{-1}$ to $A T\left(S P_{25}\right)$. Using the triangle-transitivity of $\operatorname{Tr}\left(S P_{25}\right)$, a quick computer check shows that $X$ has $5 \alpha$-neighbors in $\operatorname{Tr}\left(S P_{25}\right)$ if $X$ induces a triangle with three positive edges and $X$ has $6 \alpha$-neighbors otherwise. Notice that $\infty_{+1}$ and $\infty_{-1}$ cannot be both $\alpha$-neighbors of $X$. Thus, if $X$ does not induce three positive edges, then $X$ has at least $5 \alpha$-neighbors in $A T\left(S P_{25}\right)$. Also, if $X$ is not contained in one copy of $S P_{25}$ of the subgraph $A T\left(S P_{25}\right)$, then neither $\infty_{+1}$ nor $\infty_{-1}$ is an $\alpha$-neighbor of $X$ and thus $X$ has at least $5 \alpha$-neighbors in $A T\left(S P_{25}\right)$.

So $X$ is contained in one copy of $S P_{25}$, say $S P_{25}^{+1}$. We represent the field $\mathbb{F}_{25}$ by the numbers $x+y \sqrt{2}$, where $x$ and $y$ are integers modulo 5 . Without loss of generality, we can assume that $f\left(c_{1}\right)=0_{+1}$ and $f\left(c_{2}\right)=1_{+1}$ since $f\left(c_{1}\right) f\left(c_{2}\right)$ is a positive edge and $S P_{25}$ is arc-transitive. Moreover, $X$ induces three positive edges, so $f(b)=\beta_{+1}$ where $\beta$ is in the set $B$ of positive neighbors of 0 and 1 in $S P_{25}$. We thus have $\beta \in B=\{2,3,4,3+2 \sqrt{2}, 3+3 \sqrt{2}\}$. Table 7 gives the suitable sequences and their $4 \alpha$-neighbors in $A T\left(S P_{25}\right)$.

We are now ready to modify $f$. We decolor the vertices $a, b$, and $c$. By property $P_{2,11}$, there exist at least two colors for $b$ that are distinct from the colors forbidden by the $k$ vertices $v_{1}, \ldots, v_{k-4}, a_{1}, a_{2}, c_{1}, c_{2}$. By previous discussions, these two colors are $\beta_{+1}$ and $\beta_{+1}^{\prime}$ with $\left\{\beta, \beta^{\prime}\right\} \subset B$. Let us first set $f(b)=\beta_{+1}$. By property $P_{3,4}$, we can color $a$ such that $f(a)$ is distinct from the colors forbidden by $u_{1}, u_{2}$, and $u_{3}$. Since $f$ is not extendable to $c$ and $d$, the $4 \alpha$ neighbors of $\left(0_{+1}, 1_{+1}, \beta_{+1}\right)$ are $k_{1}, k_{2}, k_{3}$, and $f(a)$. In particular, $k_{1}, k_{2}$, and $k_{3}$ are $\alpha$-neighbors of $\left(0_{+1}, 1_{+1}, \beta_{+1}\right)$. Now we set $f(b)=\beta_{+1}^{\prime}$ and obtain that $k_{1}, k_{2}$, and $k_{3}$ are $\alpha$-neighbors of $\left(0_{+1}, 1_{+1}, \beta_{+1}^{\prime}\right)$ as well. This is a contradiction, since no two distinct sequences in Table 7 have three common $\alpha$-neighbors. 
Proof of Theorem 16. Let $(H)$ be a counterexample which is minimal with respect to $\preceq$. By Lemma 17, $H$ does not contain any of the configurations $C 1$ to $C 8$. There remains to show that every triangle-free planar graph contains at least one of these configurations. This has been already done using a discharging procedure in the proof of Theorem 2 in [10], where slightly weaker configurations were used.

Montejano et al. [8] proved that $S P_{9}$ is $\mathcal{O}_{3}$-universal, that gives $\chi_{2}(G) \leqslant 9$ whenever $G$ is an outerplanar graph. They also proved that this bound is tight (i.e. there does not exist $\mathcal{O}_{3}$-universal graph on 8 vertices). We prove here that $S P_{9}$ is the only universal target graph on 9 vertices.

Theorem 18 The only $\mathcal{O}_{3}$-universal graph of order 9 is $S P_{9}$.

Proof. We say that a 2-edge-colored path or cycle is alternating if it has an even number of edges and if every two consecutive edges have different signs. Let $(P)$ be the alternating 6-path. Note that $\chi_{2}(P)=4$. Moreover, if $(P)$ maps to a graph $T_{4}$ with 4 vertices, then $T_{4}$ contains an alternating 4 -cycle as a subgraph. Let $(H)$ be the outerplanar graph consisting in a universal vertex $u$ positively (resp. negatively) linked to every vertex of a copy $\left(P_{p}\right)\left(\operatorname{resp} .\left(P_{n}\right)\right)$ of $(P)$. So $|V(H)|=15$. Every 2-edge-colored coloring of $(H)$ uses at least 4 colors for the vertices of $\left(P_{p}\right)$, at least 4 other colors for the vertices of $\left(P_{n}\right)$, and an additional color for $u$. Therefore $\chi_{2}(H)=9$.

Let $(G)$ be the outerplanar graph obtained from 16 copies $\left(H_{0}\right),\left(H_{1}\right), \ldots$, $\left(H_{15}\right)$ of $(H)$ as follows: we identify each of the 15 vertices of $\left(H_{0}\right)$ the vertex $u$ of a copy $\left(H_{i}\right)$. Consider a graph $\left(T_{9}\right)$ with 9 vertices such that $(G)$ maps to $\left(T_{9}\right)$. In every 2-edge-colored homomorphism of $(G)$ to $\left(H_{9}\right)$, each of the nine colors appears on a vertex of $\left(H_{0}\right)$. Since a copy of $(H)$ is attached to every vertex of $\left(H_{0}\right)$, every vertex $c$ of $\left(H_{9}\right)$ satisfies $\left|N^{+}(c)\right|=\left|N^{-}(c)\right|=4$. Then, by previous discussions, we also have that both $N^{+}(c)$ and $N^{-}(c)$ contain an alternating 4-cycle.

Meringer [7] provides an efficient algorithm to generate regular graphs with a given number of vertices and vertex degree. In particular, there exist 16 4-regular graphs on 9 vertices (see Figure 8). By replacing edges by positive edges and non-edges by negative edges, we obtain the 16 2-edge-colored graphs such that every vertex has 4 positive and 4 negative neighbors. Then we can check that $S P_{9}$ (corresponding to Figure $8(n)$ ) is the only graph among these 16 graphs such that there is an alternating $C_{4}$ in both the positive and the negative neighborhood of every vertex.

Concerning planar graphs, we have that $\chi_{2}\left(\mathcal{P}_{3}\right) \geqslant 20$ [8, Corollary 9]. The following result is similar to Theorem 18 for planar graphs:

Theorem 19 If there exists an anti-twinned $\mathcal{P}_{3}$-universal graph $\left(H_{20}\right)$ of order 20, then $\left(H_{20}\right)$ is isomorphic to $\operatorname{Tr}\left(S P_{9}\right)$.

Proof. Let $(G)$ be the outerplanar graph considered in the proof of Theorem 18. Then the planar graph $\left(G^{\bullet}\right)$ maps to $\left(H_{20}\right)$ and the subgraph $(G)$ of 


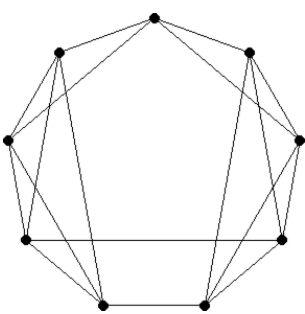

(a)

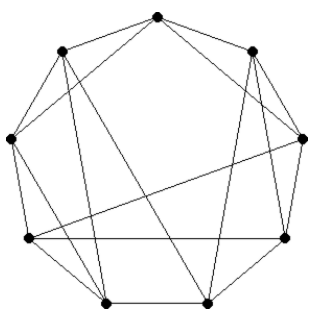

(e)

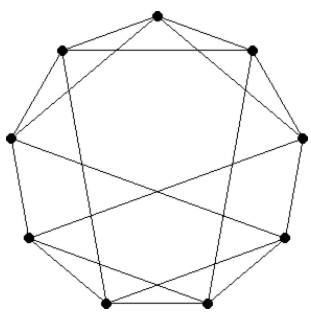

(i)

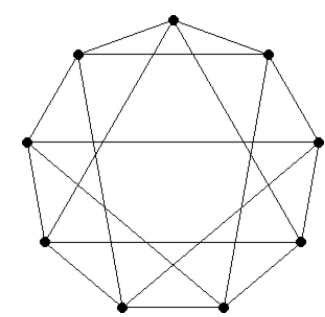

$(\mathrm{m})$

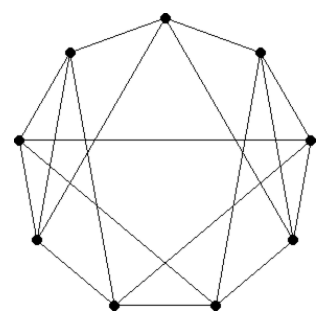

(b)

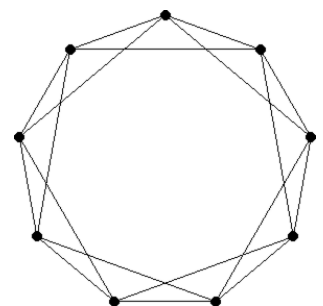

(f)

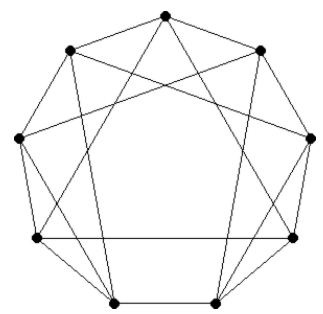

(j)

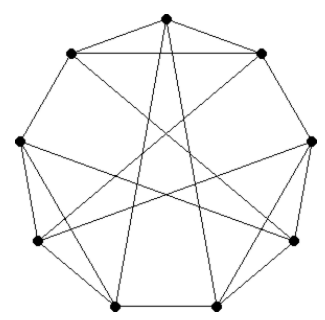

(n)

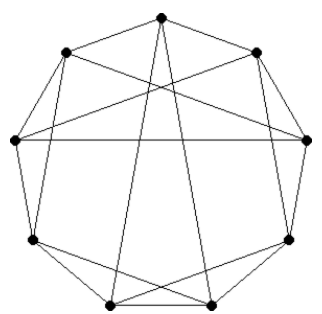

(c)

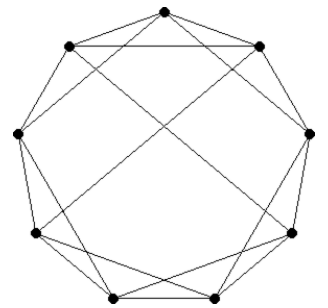

(g)

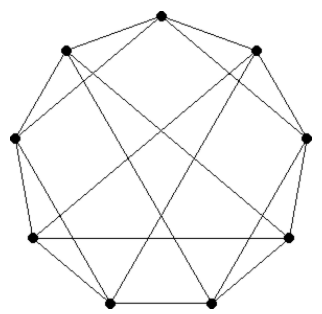

(k)

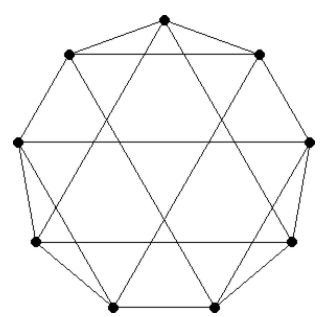

(o)

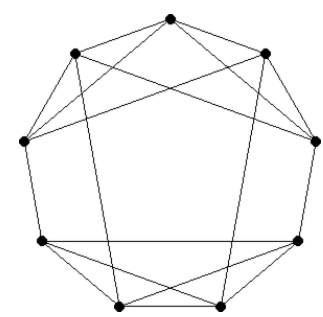

(d)

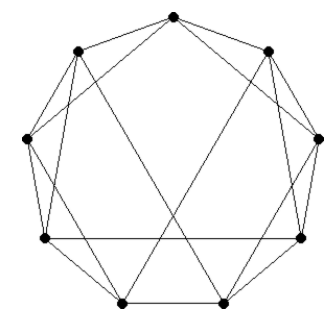

(h)

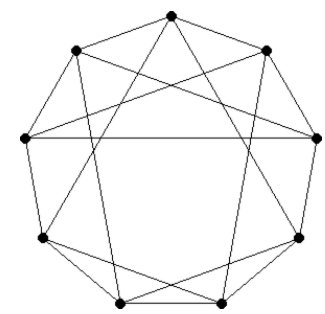

$(\ell)$

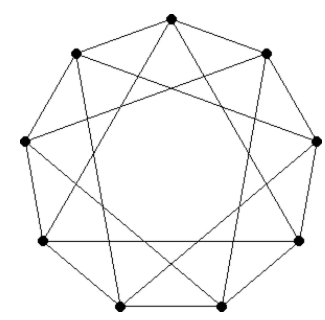

(p)

Fig. 8. The 16 connected 4 -regular graphs on 9 vertices.

$\left(G^{\bullet}\right)$ maps to the positive neighborhood of some vertex $v$ of $\left(H_{20}\right)$. Since $\left(H_{20}\right)$ is anti-twinned, every vertex has at most 9 positive neighbors. Therefore, by Theorem 18, the positive neighborhood of $v$ is isomorphic to $S P_{9}$. Then the subgraph of $\left(H_{20}\right)$ induced by $v$ and its positive neighborhood is $S P_{9}^{\bullet}$. Since $S P_{9}^{\bullet}$ is a clique of order 10 , it does not contain a pair of anti-twin vertices and thus $\left(H_{20}\right)$ is isomorphic to $A T\left(S P_{9}^{\bullet}\right)$. By the definition of 2-edge-colored Tromp Paley graphs, $\left(H_{20}\right)$ is isomorphic to $\operatorname{Tr}\left(S P_{9}\right)$.

Concerning triangle-free planar graphs, we obtain a new lower bound:

Theorem 20 There does not exist a $\mathcal{P}_{4}$-universal graph of order 11 . Thus $\chi_{2}\left(\mathcal{P}_{4}\right) \geqslant 12$. 


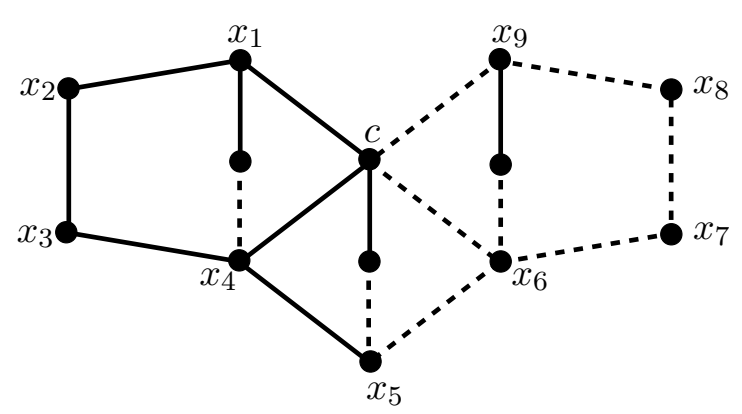

Fig. 9. The graph $(G)$ of Theorem 20.

Proof. A vertex $u$ in a 2-edge-colored graph is good if $u$ is contained in a positive cycle of odd length, in a negative cycle of odd length, and in a 4-cycle upvn such that $p u$ and $p v$ are positive and $n u$ and $n v$ are negative (these cycle are not necessarily induced). We have checked, both by hand and by computer, that:

(i) no graph with at most 5 vertices contains a good vertex,

(ii) no graph with 6 vertices contains 6 good vertices.

We consider the plane graph $(G) \in \mathcal{P}_{4}$ depicted in Figure 9. If $(G)$ admits a 2-edge-colored homomorphism $h$ to a graph $(H)$ such that the outerface of $(G)$ maps to a subgraph $\left(H^{\prime}\right)$ of $(H)$, then $h(c)$ is good in $\left(H^{\prime}\right)$. We construct the plane graph $\left(G^{\prime}\right) \in \mathcal{P}_{4}$ from 10 copies $\left(G_{0}\right),\left(G_{1}\right), \ldots,\left(G_{9}\right)$ of $(G)$ by identifying the vertex $c$ of $\left(G_{i}\right)$ with the vertex $x_{i}$ of $\left(G_{0}\right)$, for $1 \leqslant i \leqslant 9$. The embedding of $\left(G^{\prime}\right)$ is such that the $x_{j}$ 's of each of the 10 copies are on the outerface.

Suppose for contradiction that there exists a 2-edge-colored homomorphism of $\left(G^{\prime}\right)$ to a graph $(H)$ such that the outerface of $\left(G^{\prime}\right)$ maps to a subgraph $\left(H^{\prime}\right)$ of $(H)$ such that $\left(H^{\prime}\right)$ has at most 6 vertices. Since $\left(G^{\prime}\right)$ contains $(G)$ as a subgraph, $\left(H^{\prime}\right)$ contains a good vertex. So $\left(H^{\prime}\right)$ contains exactly 6 vertices by $(i)$. Moreover, every vertex on the outerface of $\left(G_{0}\right)$ corresponds to the vertex $c$ of some copy $\left(G_{i}\right)$ of $(G)$. Thus, all the 6 vertices of $\left(H^{\prime}\right)$ must be good. This contradicts $(i i)$. Therefore, if the graph $\left(G^{\prime}\right)$ maps to some graph $(H)$, then its outerface maps to some subgraph $\left(H^{\prime}\right)$ of $(H)$ of order at least 7 .

Now, we finish the proof. In a 2-edge-colored graph, two distinct vertices $u$ and $v$ are friends if $\left|N^{\alpha}(u, v)\right| \geqslant 2$ for every $\alpha \in\{-1,+1\}^{2}$. Notice that for every homomorphism $h$ of the graph $(F)$ depicted in Figure 10, the vertices $h(t)$ and $h(b)$ must be friends in the target graph. Consider a 2-edge-colored graph $(J) \in \mathcal{P}_{4}$ such that $\chi_{2}(J)=\chi_{2}\left(\mathcal{P}_{4}\right)$. We construct $\left(J^{\prime}\right)$ by adding to every vertex $u$ of $(J)$ a copy of $\left(G^{\prime}\right)$ and by connecting $u$ to every vertex on the outerface of this copy of $\left(G^{\prime}\right)$ using a copy of $(F)$. So, if $\chi_{2}\left(\mathcal{P}_{4}\right)=k$, then $\left(J^{\prime}\right)$ must map to a 2 -edge-colored clique with $k$ vertices such that every vertex has at least 7 friends. We have checked by computer that no 2-edge-colored clique with at most 11 vertices is such that every vertex has at least 7 friends.

Finally, concerning planar graphs with girth at least 7 , the following theorem gives a new lower bound: 


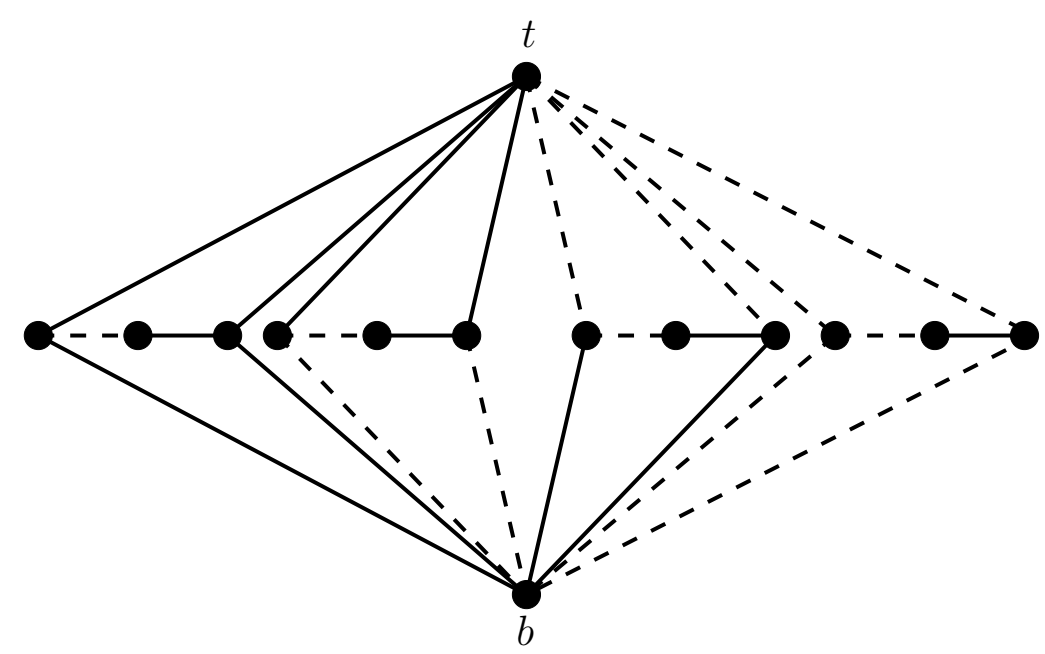

Fig. 10. The graph $(F)$ of Theorem 20 .

Theorem 21 There does not exist a $\mathcal{P}_{7}$-universal graph of order 7 . Thus $\chi_{2}\left(\mathcal{P}_{7}\right) \geqslant 8$.

Proof. Consider a 2-edge-colored graph $(J)$ such that $J \in \mathcal{P}_{7}$ and $\chi_{2}(J)=$ $\chi_{2}\left(\mathcal{P}_{7}\right)$. We construct $\left(J^{\prime}\right)$ from $(J)$ as follows. For every vertex $u$ in $(J)$ and for every pair $\left(s_{c}, s_{p}\right) \in\{-1,+1\}^{2}$, we add a 7 -cycle whose edges have sign $s_{c}$ and connect $u$ to every vertex of this 7 -cycle using a path with 3 edges of $\operatorname{sign} s_{p}$.

If $\chi_{2}\left(\mathcal{P}_{7}\right)=k$, then $\left(J^{\prime}\right)$ must map to a 2-edge-colored clique with $k$ vertices such that for every vertex $u$ and every $s_{p} \in\{-1,+1\}$, the graph induced by the vertices reachable by a walk of three edges of sign $s_{p}$ starting from $u$ contains both a positive and a negative cycle of odd length. We have checked by computer that no 2 -edge-colored clique with at most 7 vertices satisfies this property.

Table 11 summarizes the current knowledge of lower and upper bounds for the 2-edge-colored chromatic number of planar graphs with given girth.

\begin{tabular}{|c|c|c|c|c|}
\hline Girth & Lower bound & Upper bound & Target & References \\
\hline \hline$g=3$ & 20 & 80 & $S Z_{5}$ & {$[1,8]$} \\
$g=4$ & 12 & 50 & $A T\left(S P_{25}\right)$ & Th. 16, Th. 20 \\
$g=5$ & 8 & 20 & $\operatorname{Tr}\left(S P_{9}\right)$ & Th. 21, [8] \\
$6 \leqslant g \leqslant 7$ & 8 & 12 & $\operatorname{Tr}\left(S P_{5}\right)$ & Th. 21, [8] \\
$8 \leqslant g \leqslant 12$ & 5 & 8 & $S P_{9} \backslash 0$ & {$[8]$} \\
$g \geqslant 13$ & 5 & 5 & $S P_{5}$ & {$[3]$} \\
\hline
\end{tabular}

Tab. 11. Bounds on the 2-edge-colored chromatic number of planar graphs. 


\section{Results on switching homomorphism}

The graph classes considered in Theorems 12, 14, 15, and 16 admit a 2-edgecolored homomorphism to an anti-twinned graph. Using Lemma 3, we obtain good upper bounds on $\chi_{s w}$ for these classes.

Naserasr et al. [9] proved the following:

Theorem 22 ([9]) Let $G$ be a graph that admits an acyclic $k$-coloring. We have $\chi_{\text {sw }}(G) \leqslant\lceil k / 2\rceil \cdot 2^{k-1}$.

By Theorem 12, $S Z_{k}$ is $\mathcal{A}_{k}$-universal. By Proposition 5 and Lemma 3, we get the following new upper bound that improves Theorem 22 .

Theorem 23 Let $G$ be a graph that admits an acyclic $k$-coloring. We have $\chi_{s w}(G) \leqslant k \cdot 2^{k-2}$.

Recall that Huemer et al. [6] proved that Theorem 12 is tight, i.e., for every $k \geqslant 0$ there does not exist $\mathcal{A}_{k}$-universal graph of order $k \cdot 2^{k-1}-1$. In other words, there exists a graph $\left(G_{k}\right)$ that admits an acyclic $k$-coloring and such that $\chi_{2}\left(G_{k}\right)=k \cdot 2^{k-1}$. By Lemma 3 , we have that $\chi_{s w}\left(G_{k}\right) \geqslant k \cdot 2^{k-2}$, showing that Theorem 23 is actually tight. We also obtain the following results:

\section{Theorem 24}

(1) Every outerplanar graph of girth 4 admits a switching homomorphism to $S P_{5} \backslash\{0\}$. Thus, $\chi_{\text {sw }}\left(\mathcal{O}_{4}\right) \leqslant 4$.

(2) Let $(H)$ be such that $S Z_{5}=A T(H)$. Every planar graph admits a switching homomorphism to $(H)$. Thus, $\chi_{\text {sw }}\left(\mathcal{P}_{3}\right) \leqslant 40$.

(3) Every planar graph of girth 4 admits a switching homomorphism to $S P_{25}$. Thus, $\chi_{\text {sw }}\left(\mathcal{P}_{4}\right) \leqslant 25$.

(4) Every planar graph of girth 5 admits a switching homomorphism to $S P_{9}^{\bullet}$. Thus, $\chi_{s w}\left(\mathcal{P}_{5}\right) \leqslant 10$.

(5) Every planar graph of girth 6 admits a switching homomorphism to $S P_{5}^{\bullet}$. Thus, $\chi_{s w}\left(\mathcal{P}_{6}\right) \leqslant 6$.

Proof. Every statement is of the form "every graph in $\mathcal{C}$ admits a switching homomorphism to $(J)$ ". By Lemma 3, it is equivalent to the statement "every graph in $\mathcal{C}$ admits a 2-edge-colored homomorphism to $A T(J)$ ".

- Item (1) follows from Theorem 15.

- Item (2) follows from Corollary 13 (i.e., planar graphs map to $S Z_{5}$ ) and Proposition 5 (i.e., $S Z_{5}$ is anti-twinned).

- Item (3) follows from Theorem 16.

- Item (4) follows from Theorem 14(1).

- Item (5) follows from Theorem 14(2).

Concerning lower bounds, Naserasr et al. [9] constructed a planar graph $G$ such that $\chi_{s w}(G)=10$. This result also follows from $\chi_{2}\left(\mathcal{P}_{3}\right) \geqslant 20$ in [8] and Lemma 3. Moreover, we obtain the following from Theorem 19 and Lemma 3: 
Corollary 25 If every planar graph admits a switching homomorphism to a graph $\left(H_{10}\right)$ of order 10 , then $\left(H_{10}\right)$ is switching equivalent to $S P_{9}^{\bullet}$.

By Theorem 20, there exists a bipartite planar graph $(G)$ such that $\chi_{s w}(G) \geqslant$ 6 . Finally, for higher girths, note that $\chi_{s w}\left(C_{2 k}\right)=4$ for even cycles with exactly one negative edge.

\section{Conclusion}

One of our aims was to introduce and study some relevant target graphs for 2-edge-colored homomorphism. We have considered the graph $A T(G)$, the 2edge-colored Zielonka graph $S Z_{k}$, the 2-edge-colored Paley graph $S P_{q}$, and the 2-edge-colored Tromp Paley graph $\operatorname{Tr}\left(S P_{q}\right)$. Theorems 18 and 19 suggest that these target graphs are indeed interesting. Theorem 19 leads to the following question:

Open Problem 26 Is $\operatorname{Tr}\left(S P_{9}\right) \mathcal{P}_{3}$-universal?

This would imply that $\chi_{2}\left(\mathcal{P}_{3}\right)=20$ and $\chi_{s w}\left(\mathcal{P}_{3}\right)=10$. We have checked by computer that every 4 -connected planar triangulation with at most 15 vertices admits a homomorphism to $\operatorname{Tr}\left(S P_{9}\right)$. The restriction to 4-connected triangulations (i.e. triangulations without separating triangles) is justified by Lemma 8. For the $2^{25}$ non-equivalent signatures of each of the 62444 -connected planar triangulations with 15 vertices, our computer check took 150 CPUdays. Checking 4-connected triangulations with more vertices would require too much computing power.

\section{References}

[1] N. Alon and T. H. Marshall. Homomorphisms of edge-colored graphs and Coxeter groups. Journal of Algebraic Combinatorics, 8(1):5-13, 1998.

[2] O. V. Borodin. On acyclic colorings of planar graphs. Discrete Mathematics, 25:211-236, 1979.

[3] O. V. Borodin, S. J. Kim, A. V. Kostochka, and D. B. West. Homomorphisms from sparse graphs with large girth. J. Comb. Theory Ser. B, 90(1):147-159, 2004.

[4] R. Brewster and T. Graves. Edge-switching homomorphisms of edgecoloured graphs. Discrete Math., 309(18):5540-5546, 2009.

[5] B. Guenin. Packing odd circuit covers: a conjecture, Manuscript, 2005.

[6] C. Huemer, D. Flores, A. Montejano, and R. Fabila Monroy. Lower bounds for the colored mixed chromatic number of some classes of graphs. Commentationes Mathematicae Universitatis Carolinae, 49(4):637-645, 2008.

[7] M. Meringer. Fast generation of regular graphs and construction of cages. Journal of Graph Theory, 30(2):137-146, 1999.

[8] A. Montejano, P. Ochem, A. Pinlou, A. Raspaud, and É. Sopena. Homomorphisms of 2-edge-colored graphs. Discrete Applied Mathematics, 158(12):1365-1379, 2010. 
[9] R. Naserasr, E. Rollová, and É. Sopena. Homomorphisms of signed graphs. J. Graph Theory, To appear, 2014.

[10] P. Ochem and A. Pinlou. Oriented coloring of triangle-free planar graphs and 2-outerplanar graphs. Graphs and Combinatorics, 30(2):439-543, 2013.

[11] A. Pinlou and É. Sopena. Oriented vertex and arc colorings of outerplanar graphs. Inform. Process. Lett., 100(3):97-104, 2006.

[12] A. Raspaud and É. Sopena. Good and semi-strong colorings of oriented planar graphs. Inform. Process. Lett., 51(4):171-174, 1994.

[13] H. Sachs. Über selbstkomplementäre graphen. Publicationes Mathematicae Debrecen, 9:270-288, 1962.

[14] J. Tromp. Unpublished manuscript.

[15] W. Zielonka. Time-stamp systems for a fixed set of agents. Technical report, Bordeaux 1 university, 1990. 